\section{EUS-guided fine needle injection (FNI) and anti-tumor therapy}

K. J. Chang

University of California, Irvine

\section{Introduction}

EUS has evolved from EUS imaging, to EUS-guided FNA, and now to EUS-guided fine needle injection (FNI). This advancement has paved the way for "Interventional EUS" [1]. EUS-guided FNI includes delivery of contrast agents with subsequent drainage/ anastomosis, pre-EMR solution, botulinum toxin, neurolytic agents (including intra-ganglion injections), sclerosing solutions/glue, radiofrequency energy, radioactive and inert seeds, light-activating laser, and a growing number of anti-tumor agents. These will all be addressed in this update.

\section{Contrast Injection and drainage/anastomosis}

EUS-guided injection of contrast through the duodenal wall into the common bile duct was first reported by Wiersema in 1996 [2] as an alternative method for obtaining cholangiopancreatography in patients where endoscopic retrograde cholangiopancreatography (ERCP) has failed. Although technically feasible, direct injection into the bile duct or pancreatic duct for diagnostic purposes in the era of magnetic resonance cholangiopancreatography should be avoided. However, there may be special situations after failed ERCP cannulation where EUS-guided passage of a wire or EUS-guided drainage may be an alternative to percutaneous approaches. Two recent reports demonstrated the feasibility of therapeutic EUS-guided cholangiography/pancreatography with stent placement in patients with failed cannulation. Burmester et al. [3] successfully performed EUS-guided cholangio-drainage in three of four patients with malignant pancreatobiliary strictures in which ERC was not possible. They used a modification of the one-step method for performing pancreatic pseudocyst drainage described by Seifert [4]. The technique was performed by transgastric, transduodenal, and transjejunal approaches. Kahaleh et al. [5] performed EUS-guided pancreatography with gastropancreatic duct stent placement in two patients. These patients had complicated pancreatitis with pancreatic duct strictures where surgical reconstruction precluded access to the papilla. One patient developed upper gastrointestinal (UGI) bleeding 24 hours after the procedure, which was thought to be related to the needle track. This was treated endoscopically. The second patient had no procedure-related complications. Both patients had improvement in symptoms 1 month after the procedure. EUS-guided contrast injection followed by guidewire placement either through the duodenum or stomach may sal-

Correspondence: Kenneth J. Chang, M.D. · University of California, Irvine · Director, H. H. Chao Comprehensive Digestive Disease Center · 101 The City Drive, Bldg 23, Rt 81 · Orange, CA 92868 - Phone: (714) 456-6187 · Fax: (714) 4567520·E-mail: kchang@uci.edu

Bibliography: Endoscopy 2006; 38 (S1): S88-S93 (c) Georg Thieme Verlag KG Stuttgart · New York · ISSN 0013-726X · DOI 10.1055/s-2006-946664 vage difficult ERCP cannnulations by EUS-guided "rendez-vous" techniques [6]. Access and direct drainage by way of the proximal biliary tree has also been described with EUS-guided hepatico-gastrostomy $[7,8]$. These preliminary reports demonstrate that EUS-guided cholangiopancreatography with duct decompression can be successfully performed in patients where ERCP has failed. The procedure should be avoided in patients with a normal pancreas because of the potential risk of pancreatitis. Most experiences with direct injection into the pancreatic duct have been in patients with established chronic pancreatitis where the risk of iatrogenic pancreatitis is less. Larger studies with long-term follow-up are needed to fully determine the technique's safety and efficacy.

\section{Pre-EMR}

Endoscopic mucosal resection (EMR) is becoming a safe and acceptable alternative procedure for the removal of superficial cancers and complicated submucosal lesions. The ability to resect the lesion is dependent on the "lift" sign. If the lesion does not lift, this may indicate attachment to the muscularis propria, which increases the risk of perforation and lessens the chance of a curative resection. EUS has been an invaluable imaging modality to determine the depth of tumor penetration. Theoretically, if applied prior to EMR, EUS-guided injection of fluid into the precise layer under real-time ultrasound has two advantages: (1) confirmation of correct placement of the injection needle into the appropriate layer and (2) ensuring complete separation of the lesion from the normal tissue. Sun et al. [9] reported the results of 16 patients with submucosal tumors in which EMR was aided by EUS-guided saline injection. The 16 patients had lesions that were mucosal ( $n=1)$, submucosal $(n=6)$, or invading the muscularis propria $(n=9)$. They had no perforations and no recurrences at 12 to 17 months of follow-up. Whether EUS-guided injection is safer or necessary is unknown. EUS-guided injection may add to procedure time and complexity. In most cases, EUS imaging as an adjunct prior to EMR is probably sufficient.

\section{Botulinum toxin injection}

Originally reported by Pasricha et al. [10,11] endoscopic injection of botulinum toxin into the lower esophageal sphincter (LES) in patients with achalasia is safe and widely used. Initially, $90 \%$ of patients responded, but only two thirds had a sustained response at 6 months. Failure of response in some patients may be due inadequate injection into the muscle layer. EUS has the advantage of providing direct visualization of the LES, which may increase the efficacy of the procedure. Hoffman et al. [12] performed EUS-guided botulinum toxin injection in seven patients and had a sustained response, defined as no dysphagia, in six patients at a mean follow-up of 6.5 months. In a series of three patients, Maiorana et al [13] reported sustained responses. There have been no prospective randomized controlled trials comparing endoscopy versus EUS-guided injection. Due to the "field effect" of botulinum toxin injection, EUS is probably not necessary in its delivery. 


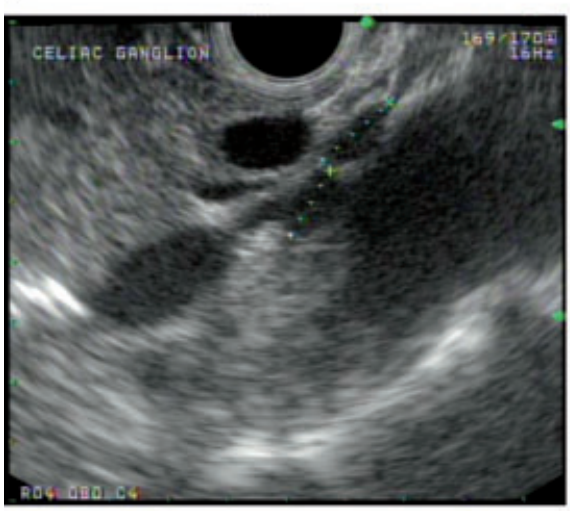

b

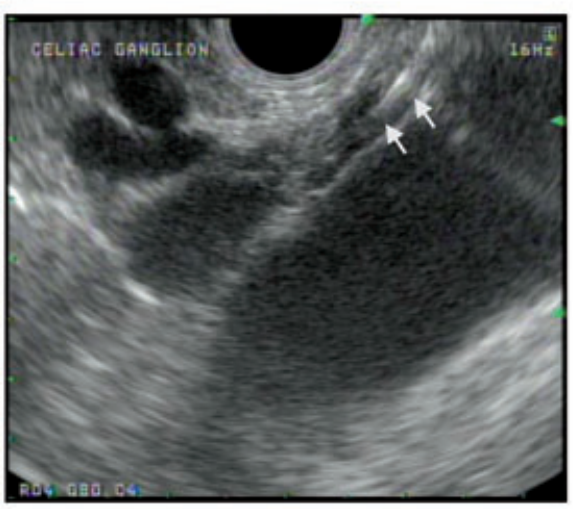

C

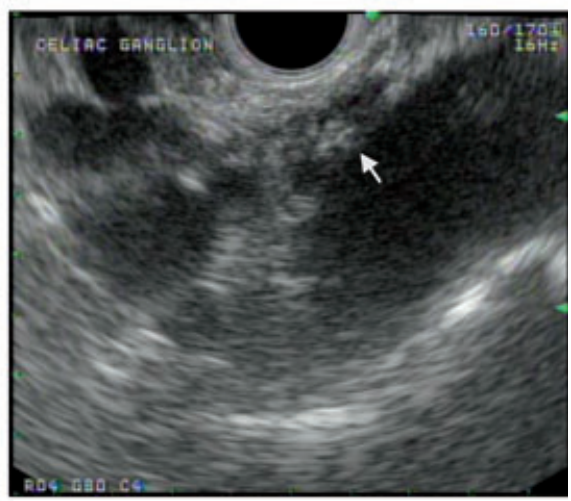

Fig. 1 a Linear EUS showing presence of 2 celiac ganglia $6.3 \mathrm{~mm}$ and $7.4 \mathrm{~mm}$ in size. b EUS-guided needle placement into ganglion for neurolysis (arrows $=$ needle within the ganglion). $\mathbf{c}$ EUS image post ganglion injection (arrow = agent in ganglion).

\section{Celiac nerve block}

Patients with significant abdominal pain who have unresectable pancreatic cancer may be candidates for EUS-guided CPN. Wiersema et al. described this novel technique and the impact on pancreatic cancer patients pain management. After visualizing the celiac trunk by the linear array echoendoscope and utilizing a 22-gauge needle, injection of bupivacaine $(0.25 \%)$ followed ethyl alcohol (98\%) can then be performed on either side of the vessel. Up to $88 \%$ of patients had persistent improvement in their pain score. Only minor complications were seen and consisted of transient diarrhea in four patients [14]. This anterior trans-gastric approach for performing CPN is theoretically considered safer when compared to the traditional CT guided posterior method. This is because of the rare reported cases of paraplegia that occurred with the posterior approach due to its proximity of the spinal column.

Unfortunately, the effect of CPN in controlling abdominal pain from chronic pancreatitis is less evident. Gress et al. performed EUS-guided CPB in 80 patients with chronic pancreatitis. A mixture of bupivacaine $0.25 \%$ and $80 \mathrm{mg}$ of triamcinolone was injected using the above-described technique. Only $10 \%$ had benefit beyond 24 weeks. There were two major complications consisted of peri-pancreatic abscess and bleeding celiac artery secondary to ethanol induced arterial pseudoaneurysm. This technique was even less effective in younger patients ( $<45$ year old) and those who had previous surgery for chronic pancreatitis [15].

Since these initial trials, most endosonographers have placed a virtual moratorium on EUS-guided CPN for patients with chronic pancreatitis due to 1 ) it's relative lack of efficacy 2) it's transient durability 3 ) it's higher complication rate and 4) possible development of retroperitoneal fibrosis (especially with alcohol injections) which may mitigate the chance for a successful islet cell transplantation (most islet cells are populated in the tail of the pancreas) as a potential future therapy. One glimmer of hope is the new appreciation that individual celiac ganglion can be visualized on EUS (personal communication with Michael Levy, Mayo Clinic Rochester, manuscript submitted) and EUS-guided "intra-ganglion" CPN can now be performed. Injection with small aliquots of alcohol directly into the ganglion may minimize the problem of retroperitoneal fibrosis and allow for subsequent follow-up injections for patients with unremitting pain from chronic pancreatitis (see Fig. 1a-c). This technique awaits further clinical trial data with guarded optimism.

\section{Cyst-gastrostomy}

Endoscopic technique for pseudocyst drainage is an established alternative for surgical and radiological approaches. Since the early report of EUS assisted pseudocyst drainage [16] the use of EUS as the only tool for draining pseudocysts has been evolving. With the development of larger accessory channels echoendoscopes this approach became feasible. EUS-guided pseudocyst drainage technique overcame many obstacles faced with the conventional endoscopic approach. In the absence of apparent intraluminal bulge, cyst drainage could still be safely performed after accurate measurement of the distance between the cyst and the gastrointestinal wall. EUS/Doppler method can be used to define and avoid any interjecting vessels. Thus, theoretically reducing the risk of hemorrhage and perforation. In cases were cystic neoplasms is of a concern, cyst fluid aspiration prior to drainage should be performed. The level of tumor markers in the fluid, mainly CEA, along with the concentration of amylase may assist in differentiating inflammatory from neoplastic cysts $[17,18]$. Giovannini et al. drained 35 pancreatic cysts under EUS-guidance [19], of whom 15 had pseudocysts and 20 had pancreatic abscesses. Of the 33 trans-gastrically drained cysts, only in 1 patient an extrinsic compression was seen using forward viewing gastroscope. No major complication occurred except in one case of a pneumoperitoneum, which was successfully managed medically. No bleeding was encountered. A 7-F nasocystic drain was placed in 18/20 cases of pancreatic abscess. Surgery was performed in the two other patients. While in the pseudocyst group, placement of an 8.5-French stent was successful in 10 patients and of a nasocystic drain in five. In one case, only cyst puncture and aspiration was performed. Over a mean follow up of 27 months (6-48 months) one recurrence among the 15 pancreatic pseudocysts and two relapses of the 18 pancreatic abscesses have been observed. The EUS-guided drainage success rate was $88.5 \%$ (31/35); only four patients with pancreatic abscesses underwent surgery. In a related paper, Seifert at el. evaluated a new 
one-step stenting device using a large channel echoendoscope $(3.2 \mathrm{~mm}$ ) for pseudocyst drainage in 6 patients [4]. One of them had pancreatic abscess. Transmural drainage was successfully done using modified 7-French diameter stents. There were no complications encountered with the endoscopic interventions. One patient with necrotizing pancreatitis, who denied surgery, died secondary to sepsis. At follow up of 3-13 months the cysts had completely resolved in four patients. This study confirmed the feasibility and effectiveness of EUS-guided one-step technique in draining various cystic lesions, however larger studies are needed. In a subsequent study and using the one-step device through a $3.7 \mathrm{~mm}$ channel echoendoscope, the same group was able to place a 10-F stent in 3 patients and 7-F in one patient, all with peripancreatic cystic lesions. One of the cysts had persisted for more than 3 months and found to be a ganglioneuroma after surgical enucleation [20].

\section{Sclerosants and glue}

Most linear array echoendoscopes are equipped with color flow mapping and Doppler ultrasound, which may be helpful in the management of UGI bleeding. A few studies have shown EUS to be helpful in targeting therapy in patients with UGI bleeding $[21,22]$. In a recent study by Lee et al. [23], 54 patients with gastric variceal bleeding underwent biweekly EUS of gastric varices followed by injection of cyanoacrylate until gastric varix obliteration was documented by the absence of anechoic vascular structures in the gastric wall. They found a statistically significant reduction $(P=0.0053)$ in rebleeding rate compared with their non-EUS control group ( $n=47$ ). Lahoti et al. [24] performed real-time EUS-guided sclerotherapy in five patients with nonbleeding esophageal varices. There were no complications, and varices were obliterated in 2.2 sessions. They concluded that the confirmation of obliteration of varices coupled with the ability to obliterate the perforating veins, which is not possible with standard sclerotherapy or banding techniques, might decrease the number of sessions and variceal recurrence. Whether secondary prevention of gastric or esophageal variceal bleeding by color flow and Doppler-assisted EUS-guided FNI improves morbidity and mortality is unknown. This application should be considered investigational and should be performed only in clinical trial settings.

\section{Radiofreqency energy and photodynamic therapy (PDT)}

Radiofrequency ablation (RFA) causes a relatively predictable zone of coagulation necrosis by intense tissue heating. Accurate and precise targeting of the tumor is important to maximize the yield and minimize morbidity to the patient. RFA is performed routinely by surgeons (laparoscopically or open) or percutaneously by ultrasound, MRI, or CT-guided methods in patients with primary, recurrent, or metastatic liver cancers. Depending on the site of the lesion, EUS may be the safest and easiest method to deliver RFA therapy. Goldberg et al. [25] published in 1999 the feasibility and effectiveness of radiofrequency ablation under EUS-guidance in 13 pigs confirmed by necroscopy. The area of necrosis measured $1 \mathrm{~cm}$. One pig had mild hyperlipasemia, a focal zone of pancreatitis $(<1 \mathrm{~cm})$, and later a pancreatic fluid col- lection. Biochemical parameters were normal in the remaining pigs. Other complications included three gastric and one intestinal burn caused by improper electrode placement. Potential applications for EUS-guided RFA may include poorly accessible liver lesions, small functional pancreatic endocrine tumors, or submucosal gastrinomas.

Photodynamic therapy (PDT) of pancreatic cancer by using percutaneously placed light catheters has been reported [26] in a group of 16 patients with advanced pancreatic cancer. All patients had substantial tumor necrosis without evidence of pancreatitis. The same Boston group [27] subsequently reported another animal experiment using EUS-guided activation of PDT to the pancreas. The animal was injected with porfimer sodium intravenously. Subsequently, a 19-gauge needle was inserted into the pancreas, the liver, the spleen, and the kidney under EUS guidance. A small diameter quartz optical fiber was passed through the EUS needle and used to illuminate the tissue with laser light. Localized tissue necrosis was achieved in all organs, without significant complication. There was no significant difference in inflammation induced by photodynamic therapy within the various organs. The advantage of PDT compared to external beam radiation for local tumor control is uncertain.

\section{Brachytherapy}

Brachytherapy, an established therapeutic modality, has been used for many years for various cancers, including head and neck, breast, lung, brain, and, most commonly, prostate cancer. Also, brachytherapy has been shown to have benefit in treating the primary esophageal tumors. A potential advantage over the more traditional external-beam radiotherapy (EBRT) is its ability to limit radiation toxicity to the normal tissues surrounding the cancer. EUS-guided brachytherapy for a primary tumor has been reported in one small series of patients with head and neck malignancy [28]. A case of EUS-guided brachytherapy in a patient with recurrent esophageal cancer in perigastric lymph nodes has recently been reported [29]. For temporary brachytherapy where the seeds are only present for a limited period of time, iridium ( Ir $^{192}$ ) is the most common isotope used. The need for hospitalization and the relatively high dose-rate delivery has made this technique less advantageous. Permanent seed brachytherapy can be done as an outpatient procedure, with little radiation safety risks. Both iodine (I ${ }^{125}$ ) and palladium (Pd ${ }^{103}$ ), which emit low-energy radiation $(<30 \mathrm{keV})$, have been used with minimal at-home precautions for patients. Though both iodine ( $\left.{ }^{125}\right)$ and palladium ( $\mathrm{Pd}{ }^{103}$ ) are suitable isotopes for permanent brachytherapy, iodine $\left({ }^{125}\right)$ may be preferable because of its relatively slower dose delivery rate (half-life of 60 days for iodine (I ${ }^{125}$ ) vs. 17 days for palladium ( $\mathrm{Pd}{ }^{103}$ ). This theoretically would result in less adjacent tissue damage, in a previously irradiated area. The adverse effects from a single seed are minimal because of low Gy emitting radiation. The size of the treated lesion determines the number of seeds used. For a large node, we may place 2 adjacent seeds. In the largest reported series of prostate brachytherapy, the percentage of migrating seeds was less than $1 \%$, with the lung as the most common site of migration, and there were no clinical consequences. A significant relationship was reported between the number of seeds implanted at the periphery 


\begin{tabular}{|c|c|c|c|c|c|c|}
\hline & \multicolumn{3}{|c|}{1 month } & \multicolumn{3}{|c|}{3 months } \\
\hline & EUS & PTA & Overall & EUS & PTA & Overall \\
\hline Tumor stabilization & $88 \%$ & $80 \%$ & $83 \%$ & $73 \%$ & $75 \%$ & $74 \%$ \\
\hline $\begin{array}{l}\text { Objective tumor response } \\
>25 \% \text { reduction in tumor area } \\
>50 \% \text { reduction in tumor area }\end{array}$ & $\begin{array}{l}38 \% \\
25 \% *\end{array}$ & $\begin{array}{r}25 \% \\
0 \%\end{array}$ & $\begin{array}{l}31 \% \\
11 \%\end{array}$ & $\begin{array}{l}33 \% \\
13 \%\end{array}$ & $\begin{array}{l}30 \% \\
10 \%\end{array}$ & $\begin{array}{l}31 \% \\
11 \%\end{array}$ \\
\hline Survival without overall progression & $56 \%$ & $68 \%$ & $63 \%$ & $53 \%$ & $42 \%$ & $47 \%$ \\
\hline
\end{tabular}

${ }^{*} \mathrm{p}=.03$, Fisher's Exact, for all other comparisons $\mathrm{p}=\mathrm{NS}$

of the prostate and the number of seeds migrating to the lungs. Therefore, placing the seed directly in the center of the node is preferable to the periphery. A group in Shanghai, China has recently published a report of ten patients with pancreatic cancer who underwent EUS-guided brachytherapy using iodine (I ${ }^{125}$ ). CT follow-up examinations were performed 1 month after the therapy. The mean number of 125 seed ranged from 4 to 12 and the activity per seed ranged from 0.4 to $0.6 \mathrm{mCi}$. Their results reported relief of pain in 9 patients after 1 to 3 days. There was a partial response in 1 case, stable disease in 6 and disease progression in 2. There were no complications reported, including pancreatitis. These 2 reports show promising preliminary data that malignant tumors in the pancreas as well as metastatic/recurrent lymph nodes can be treated safely with EUS-guided brachytherapy. Again, further studies are warranted on a larger series of patients with various tumor types and metastatic foci. In the same way that radioactive seeds can be placed by EUS guidance, inactive or inert seeds (which are radio-opaque) can be placed into various tumors and lymphnodes for the purpose of making. This may be helpful in focusing and reducing the targeted field during external beam therapy.

\section{Delivery of anti-tumor agents}

We have examined the feasibility and safety of direct injection of allogenic mixed lymphocyte culture (cytoimplant) in pancreatic adenocarcinoma under EUS guidance [30]. In a phase I clinical trial. 8 patients with unresectable pancreatic adenocarcinoma underwent EUS-guided fine needle injection (FNI) of cytoimplants. 4 patients were in stage II, 3 in stage III, and 1 in stage IV. The escalating doses of cytoimplants 3,6 , or 9 billion cells were implanted using a novel EUS guided FNI technique. The median survival was 13.2 months with 2 partial responders and 1 minor response. Major complications including bone marrow toxicity, hemorrhagic, infectious, renal, or cardiopulmonary toxicity were absent. Low-grade fever was encountered in 7 of the 8 patients, and was symptomatically treated with acetaminophen. Our study showed that local immunotherapy is feasible and safe. The technique of EUS-guided FNI was recently applied to deliver anti-tumor viral therapy [31]. ONYX-015 (dl1520) is an E1B-55kD gene-deleted replication-selective adenovirus that preferentially replicates in and kills malignant cells. Twenty-one patients with locally advanced adenocarcinoma of the pancreas or with metastatic disease, but minimal or absent liver metastases, underwent eight sessions of ONYX-015 delivered by EUS injection into the primary pancreatic tumor over 8 weeks. The final four treatments were given in combination with gemcitabine (i.v., $\left.1,000 \mathrm{mg} / \mathrm{m}^{2}\right)$. After combination therapy, 2 patients had partial regressions of the injected tumor, 2 had minor responses, 6 had stable disease, and 11 had progressive disease. No clinical pancreatitis occurred despite mild, transient elevations in lipase in a minority of patients. Two patients had sepsis before the institution of prophylactic oral antibiotics. Two patients had duodenal perforations from the rigid endoscope tip. No perforations occurred after the protocol was changed to transgastic injections only.

The most recent EUS-guided anti-tumor therapy involves a novel gene therapy [32]. TNFerade is a replication-deficient adenovector containing human TNF $\alpha$ gene, regulated by a radiation-inducible promoter Egr-1. The study design consisted of a five-week treatment of weekly intratumoral injections of TNFerade $(4 \times$ $10^{9-11}$ particle units (pu) in $2 \mathrm{~mL}$ ). EUS-guided FNI was compared to percutaneous approaches (CT or US). TNFerade was combined with continuous intravenous $5-\mathrm{FU}\left(200 \mathrm{mg} / \mathrm{m}^{2} / \mathrm{d} \times 5\right.$ days $\left./ \mathrm{wk}\right)$ and radiation (50.4 Gy). TNFerade was delivered with a single needle pass at a single site in the tumor for percutaneous approaches (PTA), while up to 4 injections were given by EUS. The clinical endpoints included safety and tumor response on spiral CT by a core lab. Of 37 pts, 17 had EUS and 20 had PTA (similar TNFerade doses). Baseline tumor stage, nodal staging, tumor size and CA 19-9 levels were similar in the EUS and PTA groups. One dose limiting toxicity (grade 3 hypotension) was noted in a PTA pt; all other adverse events potentially related to TNFerade were grade $1-2$. Procedure related adverse events were all grade 1-2 and were similar between the two groups, except for injection site pain: $35 \%$ PTA vs $0 \%$ EUS ( $p=.01$ ). There was no clinical pancreatitis. Tumor responses and disease control were similar (Table 1). Four patients underwent resection; one, a EUS patient, had a complete pathologic response. The updated report with 50 patients will have been presented at DDW 2006 [33]. At the maximal tolerated dose, $4 / 5$ patients reassessed as surgically resectable achieved pathologically negative margins, and 3 survival greater than 24 months.

EUS-guided injection of TNFerade has also been recently applied to locally advanced esophageal cancer [34]. This was a multicenter dose-escalating study of TNFerade with concurrent neoadjuvant chemoradiation $(45 \mathrm{~Gy} / 25 \mathrm{fx} / 5$ weeks, with 5 -FU [ $1000 \mathrm{mg} / \mathrm{m}^{2} /$ day x $96 \mathrm{hrs}$ ] plus cisplatin [ $75 \mathrm{mg} / \mathrm{m}^{2}$ ] on Days 1 and 29) in patients with resectable stage II and III esophageal cancer staged by CT and EUS. TNFerade was administered in 1- 
$\log$ inter-patient dose escalations from $4 \times 10^{8}-4 \times 10^{11} \mathrm{pu}$, via endoscopy $(n=18)$ or EUS $(n=6)$ once weekly x 5 weeks. Resection was performed 5-11 weeks after the end of radiation. Endpoints included maximum tolerated dose (MTD), safety and pathologic complete response rate (pCR). 24 pts were enrolled and the majority of tumors were adenocarcinoma (20/24), T3 (23/24), and N1 (18/24). The average number of injections/session was 3.9 per session. The MTD was not reached. The most frequent adverse events (AEs) judged potentially related to TNFerade were generally mild to moderate in severity and included fatigue (54\%), fever (38\%), and nausea (29\%). Thromboembolic events occurred in 8/24 (33\%) pts. In the top three dose cohorts, pCR was seen in 6/15 (40\%) of resected tumors, and 10/17 (59\%) remain disease free at a range of follow-up from 13 to 25 months. At the $4 \times 10^{9}$ pu dose, pCR was seen in $3 / 3(100 \%)$ resected tumors, with a radiographic complete response in a fourth patient; all four remain disease-free at 19 to 25 months. 20/24 pts went to surgery. The median survival has not been reached (19/24 pts are still alive) with a mean follow-up of 21 months. Comparison between endoscopy and EUS delivery showed no statistically significant differences in \# injections (3. 9 vs 3.9$)$, pCR (22\% vs $50 \%$ ), alive status ( $78 \%$ vs $83 \%$ ) or serious AE's ( $56 \%$ vs $50 \%$ ); $\mathrm{p}=$ NS. This demonstrated that endoscopy and EUS-guided FNI of TNF erade with concurrent chemoradiation is feasible and generally well-tolerated. TNFerade may result in increased pCR and optimize surgical and long-term outcomes. This represents a new treatment paradigm in esophageal cancer, with the endoscopist administering the local anti-tumor agent with real time assessment of tumor response and local toxicity.

These initial studies have established that EUS-guided FNI is an effective delivery system for anti-tumor agents both within and outside the GI lumen.

\section{Conclusions}

EUS-guided FNI has truly paved the way for therapeutic/interventional EUS. The interventional endoscopist will now play a more central role in the treatment of various GI malignancies by delivering anti-tumor agents in addition to palliating pain, relieving obstruction, and treating recurrences.

\section{References}

${ }^{1}$ Chang KJ, Wiersema MJ. Endoscopic ultrasound-guided fine-needle aspiration biopsy and interventional endoscopic ultrasonography. Emerging technologies. Gastrointest Endosc Clin N Am 1997; 7 (2): $221-235$

2 Wiersema MJ, Sandusky D, Carr R, Wiersema LM, Erdel WC, Frederick PK. Endosonography-guided cholangiopancreatography. Gastrointest Endosc 1996; 43 (2 Pt 1): $102-106$

${ }^{3}$ Burmester E, Niehaus J, Leineweber T, Huetteroth T. EUS-cholangiodrainage of the bile duct: report of 4 cases. Gastrointest Endosc 2003; 57 (2): $246-251$

${ }^{4}$ Seifert H, Dietrich C, Schmitt T, Caspary W, Wehrmann T. Endoscopic ultrasound-guided one-step transmural drainage of cystic abdominal lesions with a large-channel echo endoscope. Endoscopy 2000; 32 (3): $255-259$

${ }^{5}$ Kahaleh M, Yoshida C, Yeaton P. EUS antegrade pancreatography with gastropancreatic duct stent placement: Review of two cases. Gastrointest Endosc 2003; 58 (6): 919-923
${ }^{6}$ Mallery S, Matlock J, Freeman ML. EUS-guided rendezvous drainage of obstructed biliary and pancreatic ducts: Report of 6 cases. Gastrointest Endosc 2004; 59 (1): 100-107

${ }^{7}$ Kahaleh M, Wang P, Shami VM, Tokar J, Yeaton P. EUS-guided transhepatic cholangiography: report of 6 cases. Gastrointest Endosc 2005; 61 (2): $307-313$

${ }^{8}$ Giovannini M, Dotti M, Bories E, Moutardier V, Pesenti C, Danisi C et al. Hepaticogastrostomy by echo-endoscopy as a palliative treatment in a patient with metastatic biliary obstruction. Endoscopy 2003; 35: $1076-1078$

${ }^{9}$ Sun S, Wang M, Sun S. Use of endoscopic ultrasound-guided injection in endoscopic resection of solid submucosal tumors. Endoscopy 2002; 34 (1): $82-85$

${ }^{10}$ Pasricha PJ, Rai R, Ravich WJ, Hendrix TR, Kalloo AN. Botulinum toxin for achalasia: long-term outcome and predictors of response. Gastroenterology 1996; 110: 1410 - 1415

${ }^{11}$ Pasricha PJ, Ravich WJ, Hendrix TR, Sostre S, Jones B, Kalloo AN. Intrasphincteric botulinum toxin for the treatment of achalasia. N Engl J Med 1995; 332 (12): $774-778$

12 Hoffman BJ, Knapple W, Bhutani MS, Aabakken L, Verne N, Hawes RH. EUS-Guided Injection of Botulinum Toxin for Achalasia. Gastrointest Endosc 1996; 43 (4): A534

${ }^{13}$ Maiorana A, Fiorentino E, Genova EG, Murata Y, Suzuki S. Echo-guided injection of botulinum toxin in patients with achalasia: initial experience. Endoscopy 1999; 31 (2): 3S-4S

14 Wiersema M, Wiersema L. Endosonography guided celiac plexus neurolysis (EUS CPN) in patients with pain due to intra-abdominal malignancy (IAM). Gastrointest Endosc 1996; 43 (4): A565

15 Gress F, Schmitt C, Sherman S, Ciaccia D, Ikenberry S, Lehman G. Endoscopic ultrasound-guided celiac plexus block for managing abdominal pain associated with chronic pancreatitis: a prospective single center experience. Am J Gastroenterol 2001; 96 (2): 409-416

${ }^{16}$ Grimm H, Binmoeller KF, Soehendra N. Endosonography-guided drainage of a pancreatic pseudocyst. Gastrointest Endosc 1992; 38 (2): $170-171$

17 Brugge WR. The role of EUS in the diagnosis of cystic lesions of the pancreas. Gastrointest Endosc 2000; 52 (6 Suppl): S18 - 22

${ }^{18}$ Lewandrowski KB, Southern JF, Pins MR, Compton CC, Warshaw AL. Cyst fluid analysis in the differential diagnosis of pancreatic cysts. A comparison of pseudocysts, serous cystadenomas, mucinous cystic neoplasms, and mucinous cystadenocarcinoma. Ann Surg 1993; 217 (1): 41 - 47

${ }^{19}$ Giovannini M, Pesenti C, Rolland AL, Moutardier V, Delpero JR. Endoscopic ultrasound-guided drainage of pancreatic pseudocysts or pancreatic abscesses using a therapeutic echo endoscope. Endoscopy 2001; 33 (6): $473-477$

20 Seifert H, Faust D, Schmitt T, Dietrich C, Caspary W, Wehrmann T. Transmural drainage of cystic peripancreatic lesions with a new large-channel echo endoscope. Endoscopy 2001; 33 (12): 1022 - 1026

${ }^{21}$ Ribeiro A, Vazquez-Sequeiros E, Wiersema MJ. Doppler EUS-guided treatment of gastric Dieulafoy's lesion. Gastrointest Endosc 2001; 53 (7): $807-809$

22 Pedersen FM, Vilmann P, Bytzer P. Gastric arteriovenous malformation: Doppler EUS-guided diagnosis and therapy. Gastrointest Endosc 2002; 55 (4): 597 - 599

${ }^{23}$ Lee YT, Chan FK, Ng EK, Leung VK, Law KB, Yung MY et al. EUS-guided injection of cyanoacrylate for bleeding gastric varices. Gastrointest Endosc 2000; 52 (2): $168-174$

${ }^{24}$ Lahoti S, Catalano MF, Alcocer E, Hogan WJ, Geenen JE. Obliteration of esophageal varices using EUS-guided sclerotherapy with color Doppler. Gastrointest Endosc 2000; 51 (3): 331 - 333

25 Goldberg SN, Mallery S, Gazelle GS, Brugge WR. EUS-guided radiofrequency ablation in the pancreas: results in a porcine model. Gastrointestinal Endoscopy 1999; 50 (3): $392-401$

${ }^{26}$ Bown SG, Rogowska AZ, Whitelaw DE, Lees WR, Lovat LB, Ripley P et al. Photodynamic therapy for cancer of the pancreas. Gut 2002; 50 (4): $549-557$

27 Chan HH, Nishioka NS, Mino M, Lauwers GY, Puricelli WP, Collier KN et al. EUS-guided photodynamic therapy of the pancreas: a pilot study. Gastrointest Endosc 2004; 59 (1): 95 - 99

${ }^{28}$ Maier W, Henne K, Krebs A, Schipper J. Endoscopic ultrasound-guided brachytherapy of head and neck tumours. A new procedure for controlled application. J Laryngol Otol 1999; 113 (1): 41 - 48

${ }^{29}$ Lah JJ, Kuo JV, Chang KJ, Nguyen PT. EUS-guided brachytherapy. Gastrointest Endosc 2005; 62 (5): 805-808 
${ }^{30}$ Chang KJ, Nguyen PT, Thompson JA, Kurosaki TT, Casey LR, Leung EC et al. Phase I clinical trial of allogeneic mixed lymphocyte culture (cytoimplant) delivered by endoscopic ultrasound-guided fine-needle injection in patients with advanced pancreatic carcinoma. Cancer 2000; 88 (6): $1325-1335$

${ }^{31}$ Hecht JR, Bedford R, Abbruzzese JL, Lahoti S, Reid TR, Soetikno RM et al. A phase I/II trial of intratumoral endoscopic ultrasound injection of ONYX-015 with intravenous gemcitabine in unresectable pancreatic carcinoma. Clin Cancer Res 2003; 9 (2): 555-561

${ }^{32}$ Chang KC, Senzer N, Chung T, Hecht JR, Vogel S, Rosemurgy A et al. A novel gene transfer therapy against pancreatic cancer (TNFerade) delivered by endoscopic ultrasound(EUS) and percutaneous guided fine needle injection(FNI). Gastrointest Endosc, 2004

${ }^{33}$ Farrell JJ, Senzer N, Hecht JR, Hanna N, Chung T, Nemunaitis J, Rosemurgy A, Javle M, Reid T, Posner M, Macko J, Chang KJ. Long-term data for endoscopic ultrasound (EUS) and percutaneous (PTA) guided intratumoral TNFerade gene delivery combined with chemoradiation in the treatment of locally advanced pancreatic cancer (LAPC). Gastrointest Endosc 2006; 63 (5): AB93

${ }^{34}$ Chang KJ, Senzer N, Swisher S, Reid R, Mauer A, Posner M et al. Multicenter Clinical Trial Using Endoscopy and Endoscopic Ultrasound (EUS) guided fine needle injection (FNI) of anti-tumor agent (TNFerade $^{\mathrm{TM}}$ ) in patients with locally advanced Esophageal Cancer. Gastrointest Endosc 2006; 63 (5): AB84 